\title{
An Investigation of a Confirmed Imported Case of COVID-19 Infected Abroad - Qingpu District, Shanghai Municipality, China, November 15, 2020
}

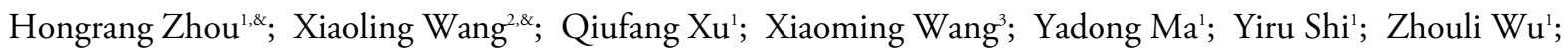 \\ Peifeng $\mathrm{Zhu}^{1}$; Jinjiang Zhao'; Ruifang $\mathrm{Xu}^{1}$; Changpo Lin ${ }^{1, * *}$; Ning $\mathrm{Xiao}^{2, *}$
}

\section{Summary \\ What is already known about this topic? \\ A passenger who was from the United States was taken to the hotel for the required isolation on November 13, 2020. During the quarantine she was diagnosed as the COVID-19 patient on November 15, 2020. Controlling the importation of COVID-19 remains a major challenge. \\ What is added by this report? \\ In this study, an epidemiological investigation was conducted for a confirmed case of COVID-19, including the treatment records in the hospital and 14- day travel trajectory before the onset of disease. \\ What are the implications for public health practice? \\ This study described an epidemiological investigation and management process on an imported case of COVID-19 and analyzed the test results, aiming to provide useful warnings to strengthen the capacity of public health system in response to the importation.}

It has been nearly 1 year since the beginning of the coronavirus disease 2019 (COVID-19) pandemic, the pathogen of which has been identified as COVID-19 virus (also known as SARS-CoV-2, 2019-nCoV, or HCoV-19) (1-2). The spread of the virus has led to a global health crisis (3). China has taken effective measures to suppress COVID-19. However, it still faces a huge challenge of detecting and dealing with imported cases (4).

This investigation was for an imported case that was detected on November 15, 2020 by Qingpu CDC, Shanghai. With the detection of this case, one of the most important tasks was an epidemiological investigation that could provide key information for screening close contacts of this case. The case was promptly sent for isolation and treatment, and the close contacts were sent to an isolated place for active and effective medical observation. The results of this investigation might benefit future surveillance efforts and mitigate the risk caused by the imported cases.

\section{INVESTIGATION AND FINDINGS}

On November 15, 2020, a Chinese traveler returned to Shanghai Municipality from the United States with symptoms such as cough, sputum, and sore throat. This patient then went to the Qingpu Branch of the Zhongshan Hospital Affiliated to Fudan University (Qingpu Zhongshan Hospital) for medical consultation. Based on the results of routine test for COVID-19, the nasal swab test was positive. Qingpu CDC subsequently used a fluorescent RT-qPCR (probe method) to verify the results. The ORF1a/b and $\mathrm{N}$ gene nucleic acid tests of COVID-19 were all positive in the nasal swab, throat swab, and sputum specimens. The case was then transferred to the Shanghai Public Health Clinical Center (SPHCC) and confirmed again.

This case occurred in an 18-year-old female international student with Chinese nationality, returning to China with her mother by flight. According to her description, she studied at a university in the United States from August 2019 to November 2020. To confirm the accuracy, we conducted separate epidemiological investigations on her and her mother from October 30 to November 13, 2020. The relevant epidemiological history of the case was shown in Figure 1.

According to the description of the patient and her mother, from October 30 to the noon of October 31, they did a yard sale at home and had contact with more than 50 people (some of the customers did not wear masks). During the period, the case only did business alone for about 1 hour (about 15-18 individuals in contact with her, half of whom did not wear masks) in the morning of October 30. Due to about 1.5 hours online lessons at home in the afternoon on the October 30, the patient did not 


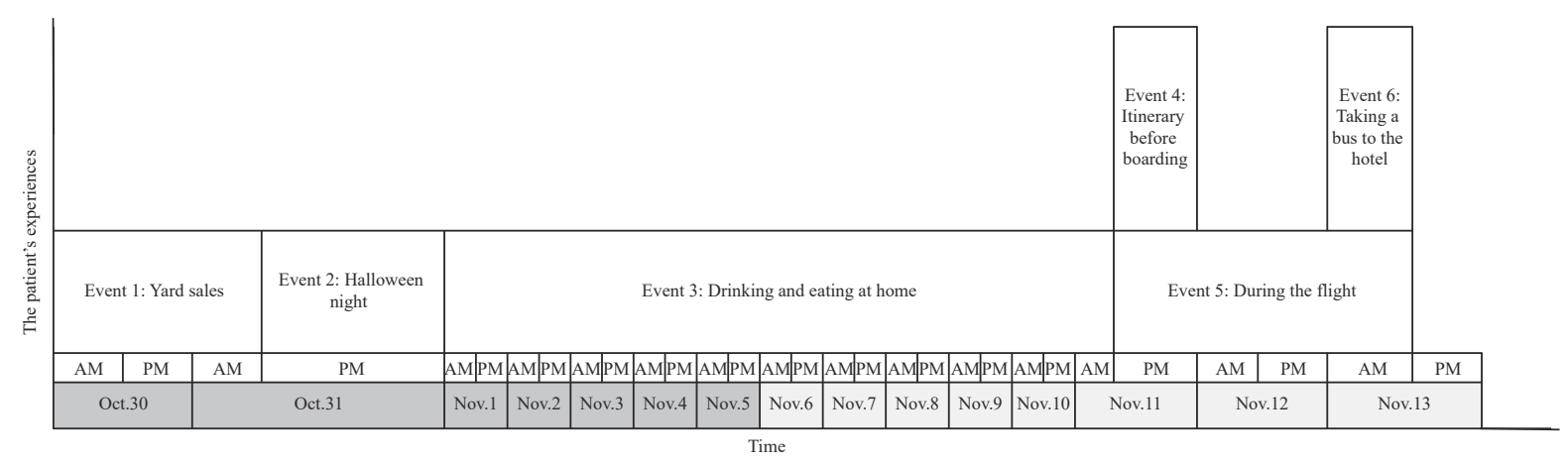

FIGURE 1. The patient's experiences during the two weeks before being detected with COVID-19 infection (according to self-reports from patient).

participate in sales. Except for the above 2 periods, both were selling together (had contact with approximately 20 people). They were close to customers (about 1 meter) every time they made sales including the sales the patient made alone. Because of Halloween, both were at home from noon to night on October 31. During the period, the case received 4 visitors (both the visitors and the patient wore masks and did not have prolonged contact with each other).

From November 1 to 11 , except for moving things to the new house, the patient and her mother were at home. Usually, the daily necessities were purchased (once a week) by an American friend who was the only person that had frequent contact during the 14-day investigation. On the afternoon of November 11, the friend drove them to the airport. Before entering the waiting hall, they had a meal at a restaurant near the airport, and there were a few customers and waiters wearing masks in the restaurant. When waiting at the terminal of the airport, the patient sat next to her mother (no people on either side of the seat, and few people around). They wore masks (except drinking water) all the time and did not go to the bathroom. However, the patient spent about 5 minutes to buy two bottles of water at the airport.

From the evening of November 11 (Pacific Standard Time) to the early morning of November 13 (Beijing Time), both the patient and her mother ate three times on the plane and drank water several times with no masks. However, the patient went to the bathroom 7-8 times (the patient reported that the bathroom environment was messy in the middle and late stages of the trip) and washed her face 1-2 times during the flight. Although the plane had stopped in Seoul City, Korea, no passengers boarded or exited. After the plane arrived in Shanghai Municipality, the patient and her mother were tested by PCR assay at the airport and took a bus with 20 other passengers who wore masks to the designated medical observation point for isolation.
The patient and her mother had undergone a series of tests. Before the patient was diagnosed, the antibody test and CT test were performed once (the first antibody test results showed that $\operatorname{IgM}$ and $\operatorname{IgG}$ were negative and the CT results were normal), and nucleic acid tests were performed four times (the result at airport customs was negative, while the results at Qingpu Zhongshan Hospital, Qingpu CDC, and the SPHCC were all positive). The test results of the patient during the detection period (performed by Qingpu Zhongshan Hospital), the treatment period (performed by SPHCC), and the 14-day isolation period (performed by Qingpu CDC) were shown in Figure 2. However, until the end of the isolation, her mother had been underwent nucleic acid tests three times (November 13, 17, and 25) and all results were negative.

\section{PUBLIC HEALTH RESPONSE}

Qingpu CDC immediately reported the case information to Shanghai CDC as soon as confirmed and the corresponding treatment measures for the patient were also actively implemented. The Shanghai CDC tracked the handling of the incident, promptly checked the patient's close contacts on the flight and soon after arriving in Shanghai. According to the rules and requirements for determining and tracking the close contacts established by the China CDC, a total of 25 close contacts (including her mother) were isolated and placed under medical observation. During the 14day quarantine, all close contacts underwent nucleic acid tests twice, and all showed negative results.

\section{DISCUSSION}

There is an incubation period in COVID-19 
infection before symptoms appear. The incubation period is normally from 1 to 14 days, mostly 3 to 7 days (5-6). After the incubation period, some infectees will show clinical symptoms. During the incubation period, the infected person has no symptoms but has a certain level of contagiousness. If appropriate isolation is not carried out, the infectee will become a potential reservoir of the infection. Therefore, during the epidemic, in order to counter the spread of COVID19 infections, the government should require all people with a clear epidemiological contact history to selfquarantine for 14 days ( 7 ).
We conducted an epidemiological investigation on the case from November 15 to 16 . Based on the selfreported travel experience of the patient and her mother and the isolation status of other passengers, we had made the following inferences about the possibility of the patient being infected with COVID-19, as shown in Table 1. Based on the investigation, we inferred that this case was most likely infected with COVID-19 during the yard sale event in the United States. The guests on Halloween night may also be a possible source of transmission. In addition, the potential risk of being infected while waiting for the

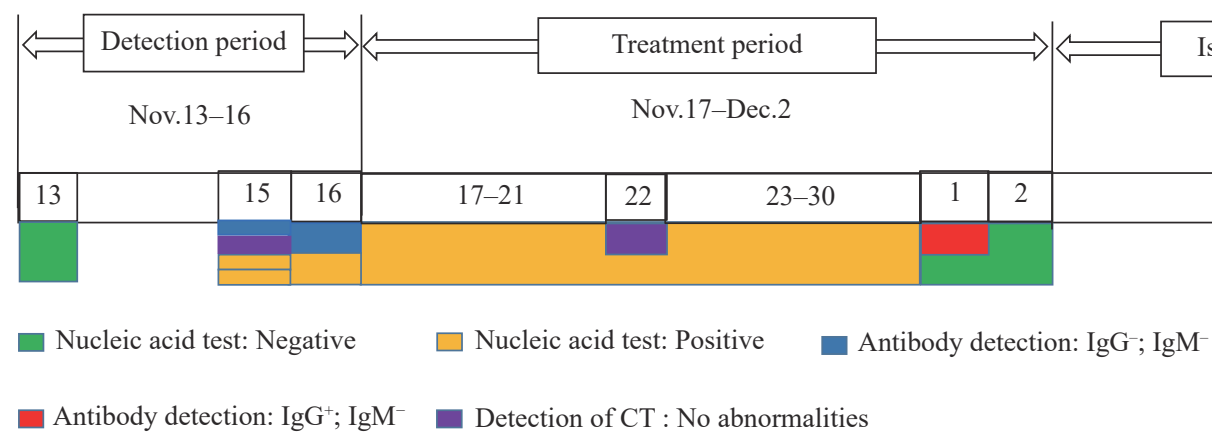

FIGURE 2. The test results of the patient during detection, treatment, and the 14-day isolation observation period in Qingpu District, Shanghai Municipality.

TABLE 1. The potential routes and risk levels of the patient's COVID-19 infection at different events during the transition from US to China.

\begin{tabular}{|c|c|c|c|}
\hline Investigation & Events & Reason & Risk level \\
\hline \multirow{6}{*}{$\begin{array}{c}\text { First } \\
\text { investigation* }\end{array}$} & 1 & $\begin{array}{l}\text { The patient contacted many customers alone for about 3-4 minutes and half of the } \\
\text { customers did not wear a mask, but the patient wore a mask. }\end{array}$ & Extremely high \\
\hline & 2 & $\begin{array}{l}\text { The patient entertained } 4 \text { visitors alone during Halloween and opened the door twice. } \\
\text { But the visitors did not enter the door and both the patient and the visitors wore masks. }\end{array}$ & Medium \\
\hline & 3 & Neither the patient's mother nor American friends had symptoms. & Low \\
\hline & 4 & $\begin{array}{l}\text { The patient basically stayed with the mother and wore masks, but she spent about } 5 \\
\text { minutes buying } 2 \text { bottles of water when waiting for the flight. }\end{array}$ & Medium \\
\hline & 5 & $\begin{array}{l}\text { The patient went to the bathroom about } 7-8 \text { times and took off the mask } 1-2 \text { times in the } \\
\text { bathroom to wash her face. The patient reported that the bathroom environment was } \\
\text { messy in the later stages of the flight. }\end{array}$ & High \\
\hline & 6 & $\begin{array}{l}\text { All passengers had no abnormal symptoms. The patient and other passengers wore } \\
\text { masks. }\end{array}$ & Low \\
\hline \multirow{6}{*}{$\begin{array}{l}\text { Follow-up } \\
\text { investigation }^{\dagger}\end{array}$} & 1 & As mentioned in the first investigation. & Extremely high \\
\hline & 2 & As mentioned in the first investigation. & Medium \\
\hline & 3 & $\begin{array}{l}\text { End of the 14-day quarantine period, the mother's nucleic acid test was negative. The } \\
\text { friend had not shown any signs. }\end{array}$ & Very low \\
\hline & 4 & As mentioned in the first investigation. & Medium \\
\hline & 5 & $\begin{array}{l}\text { The nucleic acid tests of other passengers on the flight were negative. After the 14-day } \\
\text { quarantine period, no other positive cases of the flight. }\end{array}$ & Very low \\
\hline & 6 & $\begin{array}{l}\text { After the 14-day quarantine period, no other positive cases were found among the } \\
\text { passengers on the bus }\end{array}$ & Very low \\
\hline
\end{tabular}

*The basis for judgment: the epidemiological survey report from November 15 to 16.

${ }^{\dagger}$ The basis for judgment: on November 27, the passengers of this flight had met the 14-day quarantine and were discharged. 
flight cannot be ruled out. The risk of infection for other passengers on the flight increased due to the presence of asymptomatic infections. The possibility of infection in the aircraft could also be nonnegligible depending on the flight range, in which a longer period of flight would increase the risk of transmission. For example, the toilets on the aircraft become humid and messy when used by many people, which might create conditions for the transmission. The toilet space on the plane was slightly narrow, which greatly increased the risk of infection during the prolonged flight. The investigator stated that she had visited the bathroom many times during the flight. In this investigation, all the other passengers on the flight were not positive during the 14-day quarantine period, and there had been no reports of related infections since then. However, this reminds us that the "toilet incident" might be a loophole of prevention and control on the airplane that is worth taking appropriate countermeasures for. For example, disinfection supplies should be equipped in the toilets of the aircraft and warning signs should be posted in the corresponding places to remind passengers to disinfect appropriately.

The laboratory diagnosis of this case indicated that comprehensive judgment based on clinical and test results was important. Except for nucleic acid detection, the detection of antibodies (8) and CT scans (9) played an important role in the determination of COVID-19. The first two tests for antibodies (IgG and $\operatorname{IgM})$ of the patient were negative, which suggested that the patient was in an early stage of onset. The results of the first CT scan could also be verified accordingly. However, the positive test results of the ORF $1 \mathrm{a} / \mathrm{b}$ and $\mathrm{N}$ genes of COVID-19 in the sputum specimen of this patient indicated that the subject had the ability to spread the virus. On the seventh day in the SPHCC, the results of the CT scan of the patient were also normal, which might be due to early case detection and treatment. In addition, monitoring and management during isolation were also of great importance. The patient recovered after 17 days of treatment. In order to ensure safety, the subject was quarantined for another 14 days. This person has since been discharged from isolation.

So far, COVID-19 is still raging around the world. With the large-scale resumption of work and production of Chinese enterprises, the prevention and control of COVID-19 are still facing huge challenges from imported cases and locally sporadic cases caused by the imported cases or cold chain transport (10). An efficient multisectoral collaboration mechanism and a sensitive surveillance and response system will greatly contribute to prevention and control of the COVID-19.

doi: $10.46234 / \mathrm{ccdcw} 2021.061$

\# Corresponding authors: Changpo Lin, linchangpo@163.com; Ning Xiao,xiaoning@nipd.chinacdc.cn.

\footnotetext{
Qingpu District Center for Disease Control and Prevention, Shanghai, China; ${ }^{2}$ National Institute of Parasitic Diseases, Chinese Center for Disease Control and Prevention, Shanghai, China; ${ }^{3}$ Program in Public Health, College of Health Sciences, University of California at Irvine, Irvine, USA.

${ }^{\&}$ Joint first authors.
}

Submitted: January 04, 2021; Accepted: March 02, 2021

\section{REFERENCES}

1. Jiang SB, Shi ZL, Shu YL, Song JD, Gao GF, Tan WJ, et al. A distinct name is needed for the new coronavirus. Lancet 2020;395(10228):949. http://dx.doi.org/10.1016/S0140-6736(20)30419-0.

2. Zhu N, Zhang DY, Wang WL, Li XW, Yang B, Song JD, et al. A novel coronavirus from patients with pneumonia in China, 2019. N Engl J Med 2020;382(8):727 - 33. http://dx.doi.org/10.1056/NEJMoa2001 017.

3. Sohrabi C, Alsafi Z, O'Neill N, Khan M, Kerwan A, Al-Jabir A, et al. World Health Organization declares global emergency: a review of the 2019 novel coronavirus (COVID-19). Int J Surg 2020;76:71-6. http://dx.doi.org/10.1016/j.ijsu.2020.02.034

4. Pan JH, Tian J, Xiong HY, Liu ZX, Yao Y, Wang YS, et al. Risk assessment and evaluation of China's policy to prevent COVID-19 cases imported by plane. PLoS Negl Trop Dis 2020;14(12):e0008908. http://dx.doi.org/10.1371/journal.pntd.0008908.

5. Lauer SA, Grantz KH, Bi QF, Jones FK, Zheng QL, Meredith HR, et al. The incubation period of coronavirus disease 2019 (COVID-19) from publicly reported confirmed cases: estimation and application. Ann Intern Med 2020;172(9):577 - 82. http://dx.doi.org/10.7326/ M20-0504.

6. McAloon C, Collins Á, Hunt K, Barber A, Byrne AW, Butler F, et al. Incubation period of COVID-19: a rapid systematic review and metaanalysis of observational research. BMJ Open 2020;10(8):e039652. http://dx.doi.org/10.1136/bmjopen-2020-039652.

7. Shi QL, Hu YY, Peng B, Tang XJ, Wang W, Su K, et al. Effective control of SARS-CoV-2 transmission in Wanzhou, China. Nat Med 2021;27(1):86 - 93. http://dx.doi.org/10.1038/s41591-020-01178-5.

8. Zhong L, Chuan JL, Gong B, Shuai P, Zhou Y, Zhang Y, et al. Detection of serum IgM and IgG for COVID-19 diagnosis. Sci Chin Life Sci 2020;63(5):777 - 80. http://dx.doi.org/10.1007/s11427-0201688-9.

9. Tenda ED, Yulianti M, Asaf MM, Yunus RE, Septiyanti W, Wulani V, et al. The importance of chest CT scan in COVID-19. Acta Med Indones 2020;52(1): 68-73. https://pubmed.ncbi.nlm.nih.gov/322 91374/.

10. Zhao X, Mao LL, Zhang JQ, Zhang Y, Song Y, Bo ZJ, et al. Reemergent cases of COVID-19 - Dalian City, Liaoning Province, China, July 22, 2020. China CDC Wkly 2020;2(34):658-60. http://dx.doi.org/10.46234/ccdcw2020.182. 